\title{
Staphylococcus aureus: The persistent pathogen
}

\author{
B Lynn Johnston $\mathrm{MD}^{1}$, John M Conly MD²
}

$I^{\prime}$ In the last issue of this journal, the recent emergence of community-acquired methicillin-resistant Staphylococcus aureus (MRSA) was reviewed (1). Even with all of the attention paid to the increasing prevalence of methicillin resistance, it is instructive to reflect on the importance of $S$ aureus as a pathogen, regardless of antimicrobial susceptibility, and on the management of $S$ aureus bacteremia.

Laupland et al (2) recently examined the epidemiology of invasive $S$ aureus (ISA) infections in a population-based surveillance study within the Calgary Health Region from May 1999 to May 2000. The incidence of ISA infection was 28.4 cases $/ 100,000$ persons, with an incidence rate for bacteremia of 17.9 cases/100,000 persons. Of the invasive infections, $46 \%$ were nosocomial, and $24 \%$ of community-acquired infections were health care-related. Only seven of the 264 patients were infected with MRSA. Patient groups at risk for ISA were those undergoing hemodialysis or peritoneal dialysis; diabetics; injection drug users; alcoholics, and those with HIV; heart disease; chronic obstructive pulmonary disease; stroke; cancer and solid organ transplants. These groups may simply reflect patients with high intensity of exposure to the health care system and instrumentation. The case fatality rate was $19 \%$. Independent risk factors for death in multivariable analysis were bacteremia, respiratory focus of infection, receipt of empiric antimicrobials within $8 \mathrm{~h}$, older age and hypotension. The authors speculated that receipt of empiric antimicrobials within $8 \mathrm{~h}$ was a marker for a patient with a greater severity of illness and an inherent higher risk for death.

Similar findings were reported by Morin and Hadler (3). In a 1998 population-based study of $S$ aureus bacteremia in Connecticut, the incidence rate was 17 cases/100,000 persons; just slightly lower than the pneumococcal bacteremia rate of 21.2 cases/100,000 persons. Over half $(52 \%)$ of the 402 cases were hospital-acquired and $62 \%$ of the community-acquired cases had some type of contact with the health care system in the previous year. In the remaining community-acquired cases, $85 \%$ of the patients had an underlying medical condition. The case fatality rate was $11 \%$, lower than that reported in most studies.

Evidence suggests that the rates of nosocomial and communityacquired $S$ aureus bacteremia have risen over time, no doubt related to the increasing complexity of patient problems and the invasive devices used in providing care. In a 12-year study, staphylococci were the leading pathogens of nosocomial bacteremia at the University of Iowa Hospitals and Clinics (4). In that setting, $S$ aureus was one of four pathogens accounting for the increase in overall bloodstream infection rate from 6.7/1000 discharges in 1980 to $18.4 / 1000$ discharges in 1992 . A hospital-based study was conducted in Atlanta to compare bloodstream infections due to $S$ aureus during 1980 to 1983 and 1990 to 1993 (5). Steinberg et al (5) found that the rate of nosocomial $S$ aureus bacteremia and community-acquired bacteremia increased from 0.75 cases $/ 1000$ discharges to 2.80 cases/1000 discharges and from 0.84 cases/1000 discharges to 2.43 cases/1000 discharges, respectively, between the two time frames. Additional observations from this study were an eight-fold increase in the number of nosocomial device-related infections (50\% of bacteremias during 1990 to 1993) and the increasing role of devices in community-acquired bacteremias (0\% in 1980 to 1983 and 22\% in 1990 to 1993 ). Sources of community-acquired device-related bacteremias during 1990 to 1993 were implanted ports $(49 \%)$, hemodialysis catheters $(33 \%)$, tunneled cuffed catheters $(9 \%)$ and peripheral lines (5\%) (5).

A number of studies have examined the clinical features of $S$ aureus bacteremia (SAB). In a population-based study designed to evaluate outcomes of SAB in Denmark, Jensen et al (6) described the epidemiology of SAB and contrasted the features of hospital- and community-acquired cases. The incidence rate was 1.18 cases/1000 admissions, of which $53 \%$ (147 cases) were hospital-acquired and 47\% (131 cases) were community-acquired. Patients with community-acquired SAB were older, more likely to be injection drug users, and more likely to have skin or an unknown focus, endocarditis and osteomyelitis. The overall mortality rate was $34 \%$ and trended toward being higher in patients with community-acquired SAB. The mortality rate was higher in patients with septic shock and lower in hemodialysis patients, but not associated with the type of primary or secondary focus or presence or absence of secondary infection.

In a seven-year study of hospitalized patients with SAB in Switzerland during the 1980s, 200 of 281 (71\%) cases were hospital-acquired (7). Patients with hospital-acquired SAB were more likely to have an intravascular device, surgical wound, previous hospitalization, alcohol abuse, trauma, an apparent primary site of infection, and a removable focus of infection than patients with community-acquired SAB. The overall mortality was $33.6 \%$, with no significant difference between hospitaland community-acquired cases. However, a higher mortality rate was observed in patients with complicated bacteremia, primary bacteremia, or endocarditis. In a comparison of SAB

${ }^{1}$ Queen Elizabeth II Health Sciences Centre and Dalhousie University, Halifax, Nova Scotia; ${ }^{2}$ Departments of Pathology and Laboratory Medicine, Medicine, and Microbiology and Infectious Diseases, University of Calgary, Calgary, Alberta

Correspondence: Dr Lynn Johnston, Department of Medicine, Room 5014 ACC, Queen Elizabeth II Health Sciences Centre, 1278 Tower Road, Halifax, Nova Scotia B3H 2Y9. Telephone 902-473-5553, fax 902-473-7394, e-mail ljohnsto@dal.ca 
among elderly (66 to 90 years) and younger (18 to 60 years) individuals, mortality was higher in the elderly, even after adjusting for confounding variables (8). Of note in this study, however, was that MRSA bacteremia was more common in the elderly, perhaps contributing to the higher mortality.

More recently, Mylotte and Tayara (9) reviewed SAB between January 1995 and February 1999 at a Buffalo medical centre. Patients with hospital- and community-acquired SAB had similar portals of entry, perhaps reflecting the increased movement of health care and its attendant risks into the community setting. Overall mortality was $28 \%$, with no difference between community- and hospital-acquired cases. The most important predictors of mortality were an acute physiology score of at least 60 at onset of SAB, unknown focus of infection and a respiratory focus.

The frequency of complications from SAB ranges from 11\% to $53 \%$ (10). These complications vary from endocarditis to metastatic foci and include the problem of relapse. In their study of community-acquired SAB in 113 noninjection drug users, Willcox et al (11) found that $32 \%$ of patients developed acute renal failure and $29 \%$ had adult respiratory distress syndrome. There were 19 patients with infective endocarditis, for whom the mortality was $68 \%$, compared with $35 \%$ overall for patients with SAB. As we see more cases of device-related $\mathrm{SAB}$, this becomes an issue of increasing concern. Sanabria et al (12) reported that $S$ aureus accounted for $40 \%$ of endocarditis cases between 1981 and 1986, compared with 20\% to $25 \%$ of cases three decades earlier. None of the cases was reported to be due to an intravascular catheter. In contrast, Watanakunakorn (13) found that $17 \%$ of 106 cases of S aureus endocarditis diagnosed at a community hospital in Ohio were nosocomial, with 15 of 18 cases associated with infected intravascular catheters. Of note was that 26 patients had no heart murmur and a normal transthoracic echocardiogram. The overall in-hospital mortality was $25.5 \%$. In a study of 59 patients with $S$ aureus endocarditis prospectively identified at Duke University Medical Centre from September 1994 to January 1998, 45.8\% of the patients had hospital-acquired SAB (14). The presumed source of infection was an intravascular device in $50.8 \%$ of patients. Fifteen patients $(25.4 \%)$ developed infective endocarditis (IE) after surgical procedures, and in 14 patients, a surgical wound was the presumed source. The crude mortality rate was $22 \%$.

Finally, in a Danish population-based study from 1982 to 1991, 260 patients with $S$ aureus endocarditis were identified (15). S aureus IE accounted for $2.3 \%$ to $3.7 \%$ of the total number of SABs occurring during the study period. Eighty-seven cases $(33 \%)$ were hospital-acquired. In these nosocomial cases, the median duration from admission to onset of disease was 12 days. Of note is that the clinical presentation was often nonspecific, with a new heart murmur in only $31 \%$ at presentation. Overall, the diagnosis of IE was not suspected clinically in 83 cases $(32 \%)$. Undiagnosed cases were older patients, and less frequently had a new heart murmur and predisposing heart condition. Additionally, undiagnosed cases more often had a primary focus of infection. The mortality rate in clinically diagnosed patients was $46 \%$, higher in those 60 years of age or older, or with late congestive heart failure or central nervous system involvement.

Looking more specifically at complications of nosocomial bacteremia in patients with prosthetic heart values, Fang et al (16) determined that $43 \%$ of such patients had prosthetic valve endocarditis (PVE). Fifty-six (33\%) had PVE at the time the bacteremia was discovered, while 18 developed endocarditis a mean of 45 days later. $S$ aureus accounted for 34 of 171 bacteremias and 15 of 74 cases of IE. Staphylococcal infection occurred more frequently in patients developing new endocarditis than in patients with bacteremia not complicated by endocarditis. The 12-month mortality for PVE was $47 \%$.

Given the high mortality associated with SAB, treatment becomes an important issue. However, there is little prospective data defining the optimal agents and duration of therapy for SAB, including endocarditis. The generally recommended duration of therapy for invasive, life-threatening infections, including endocarditis, osteomyelitis or arthritis, is at least four weeks (10). Recent endocarditis treatment guidelines recommend four to six weeks of a semisynthetic penicillin, with or without the addition of an aminoglycoside for the first three to five days (17). Shorter (14-day) course regimens of a semisynthetic penicillin and gentamicin are suggested for certain cases of right-sided endocarditis (17). PVE and cases due to MRSA require special consideration. The clinical evidence on which these recommendations are based is limited, with much of the supporting data coming from in vitro susceptibility testing and animal endocarditis models (18). In the one randomized trial of treatment of $S$ aureus endocarditis, 48 intravenous drug users (IVDU) and 30 non-IVDU were randomized to receive nafcillin for six weeks, with or without gentamicin for two weeks (19). The combined therapy did not alter mortality or morbidity in either group, but did result in a more rapid clinical response (duration of fever and normalization of leukocyte count), and reduced duration of bacteremia in patients with right-sided endocarditis. Observational studies comparing patients who received combination therapy (semisynthetic penicillin or first generation cephalosporin or vancomycin plus aminoglycoside and/or rifampin) have failed to demonstrate a mortality benefit to combination therapy $(11,15,20)$.

There have been several randomized trials comparing treatment options for right-sided endocarditis or endocarditis in IVDU, most with isolated right-sided involvement. From August 1976 to December 1977, 25 episodes of S aureus IE in IVDU were randomized to a beta-lactam antibiotic for four weeks, with or without gentamicin for the first two weeks (21). There were no deaths in either treatment group and time to defervescence was equal. In a noncomparative study of 53 episodes of right-sided IE in IVDU (22), nafcillin or vancomycin plus tobramycin for 14 days was associated with a 94\% cure rate at time of discharge, with the nafcillin combination performing better than vancomycin (33\% cure). Ribera et al (23) subsequently determined in a randomized, controlled trial of right-sided IE treatment in 74 IVDU that there was no benefit to the addition of gentamicin to a two-week course of cloxacillin. More recently, Fortún et al (24) demonstrated that a short course of a glycopeptide and gentamicin is inferior to cloxacillin and gentamicin for the treatment of $S$ aureus rightsided endocarditis. These trials have generally been very selective in the patients enrolled, excluding complicated patients as defined by: isolation of MRSA, beta-lactam allergies, extrapulmonary metastatic foci, renal insufficiency, pregnancy, leftsided endocarditis and mechanical valves $(23,24)$.

The appropriate duration of therapy for bacteremia originating from an intravascular catheter, in the absence of endocarditis or a metastatic focus, is controversial (10). In a three-month follow-up of 13 patients with $S$ aureus catheter- 
associated bacteremia (CAB), treated intravenously for an average of 12 days, Ehni and Reller (25) found that only one patient had relapsed bacteremia after having received nine days of intravenous antibiotics. Based on their results and a review of the literature, they suggested that short-course therapy for uncomplicated $S$ aureus $\mathrm{CAB}$ was reasonable. In a study of 55 patients with catheter-related $S$ aureus bacteremia at the University of Florida's affiliated hospitals (26), multivariable analysis showed that three of 28 (17\%) patients treated for less than 10 days relapsed with septicemia, compared with zero of 28 patients treated for longer than 10 days $(\mathrm{P}=0.05)$. A delayed clinical response to removal of the catheter and initiation of antibiotics was associated with acute early complications. The authors recommended that a two-week course of antibiotic therapy should be given to patients with no underlying valvular disease who respond rapidly to treatment, but that patients who respond slowly may require a course of intravenous therapy exceeding two weeks. In an effort to determine the effectiveness of short-course antibiotic therapy for $S$ aureus CAB, Jernigan and Farr (27) performed a systematic review of English-language publications. They identified only 11 studies, potentially flawed by both bias and statistical imprecision, reporting late complication rates of $0 \%$ to $29 \%$ (pooled estimate $6.1 \%$ ), regardless of duration of therapy. Malanoski et al (28) noted that two of three patients treated for less than 10 days for $S$ aureus CAB had relapse, whereas, there were no differences in relapse rates for those treated 10 to 15 days compared with longer courses. While a two-week period of therapy has generally been recommended for infections considered to pose a low risk of complications $(10,26)$, even this approach comes with cautions regarding its adequacy $(27,29)$. Some of the late relapses may be due to unrecognized endocarditis in patients with $S$ aureus bacteremia. This has led to the recommendation to perform transesophageal echocardiography in all patients with S aureus bacteremia $(11,14,15,30,31)$.

Several observational studies have identified predictors of outcome for $S$ aureus bacteremia. In an evaluation of outcomes in patients with nosocomial $S$ aureus bacteremia, delay in receiving appropriate therapy (breakpoint of $44.75 \mathrm{~h}$ on

\section{REFERENCES}

1. Conly JM, Johnston BL. The emergence of methicillin-resistant Staphylococcus aureus as a community-acquired pathogen in Canada. Can J Infect Dis 2003;14:249-51.

2. Laupland KB, Church DL, Mucenski M, et al. Populationbased study of the epidemiology of and the risk factors for invasive Staphylococcus aureus infections. J Infect Dis 2003;187:1452-9.

3. Morin CA, Hadler JL. Population-based incidence and characteristics of community-onset Staphylococcus aureus infections with bacteremia in 4 metropolitan Connecticut areas, 1998. J Infect Dis 2003;184:1029-34.

4. Pittet D, Wenzel RP. Nosocomial bloodstream infections: Secular trends in rates, mortality, and contribution to total hospital deaths. Arch Intern Med 1995;155:1177-84.

5. Steinberg JP, Clark CC, Hackman BO. Nosocomial and communityacquired Staphylococcus aureus bacteremias from 1980 to 1993: Impact of intravascular devices and methicillin resistance. Clin Infect Dis 1996;23:255-9.

6. Jensen AG, Wackmann CH, Espersen F, et al. Treatment and outcome of Staphylococcus aureus bacteremia: A prospective study of 278 cases. Arch Intern Med 2002;162:25-32.

7. Lautenschlager S, Herzog C, Zimmerli W. Course and outcome of bacteremia due to Staphylococcus aureus: Evaluation of different clinical case definitions. Clin Infect Dis 1993;16:567-73. regression analysis) carried a 3.8 times higher mortality and was associated with a longer hospital stay (32). A study examining adherence to treatment guidelines for $S$ aureus bacteremia detected a higher relapse rate, but no difference in mortality, in patients who received inadequate therapy (33). Insufficient treatment duration was the most common reason for inadequate treatment. In their prospective study of 278 cases of $\mathrm{S}$ aureus bacteremia, Jensen et al (6) found that presence of an uneradicated focus, total daily dose of semisynthetic penicillin of less than $4 \mathrm{~g}$, and duration of treatment of less than 14 days, were risk factors for death, with the presence of a secondary focus and daily semisynthetic penicillin dose of less than $3 \mathrm{~g}$ being predictive of relapse. Fowler et al (34) in a prospective evaluation of the management of 244 hospitalized patients with $S$ aureus bacteremia, found that patients whose intravascular devices were not removed were 6.5 times more likely to relapse or die from infection than were those whose devices were removed. They also found that patients for whom the recommendations of an infectious diseases specialist were followed were more likely to be cured of their $S$ aureus infection and less likely to relapse than patients for whom recommendations were not followed.

In summary, ISA infections are common $(2,3)$. Most of these infections are bacteremias, with hospital-acquired cases responsible for a considerable proportion $(2,3,6,7,9)$. Infective endocarditis, including prosthetic valve endocarditis, is a recognized complication and may not be diagnosed clinically $(11,13-16)$. Thus, a high index of suspicion and greater use of echocardiography are required for a timely diagnosis $(11,14,15,30,31)$. Clinical evidence does not demonstrate improved outcomes with the use of combination therapy where prosthetic values are not involved $(11,15,19-21,23)$, but suggests that glycopeptides may be inferior to beta-lactams for methicillin-susceptible strains $(22,24)$. Effective management requires an adequate course of antimicrobials (agent, duration, dose and prompt initiation) as well as the timely removal of removable foci of infection (6,26,32-34). The optimal duration of therapy for uncomplicated $\mathrm{S}$ aureus $\mathrm{CAB}$ remains to be determined $(10,26,27,29)$.

8. McClelland RC, Fowler VG, Sanders LL, et al. Staphylococcus aureus bacteremia among elderly vs younger adult patients: Comparison of clinical features and mortality. Arch Intern Med 1999;159:1244-7.

9. Mylotte JM, Tayara A. Staphylococcus aureus bacteremia: Predictors of 30-day mortality in a large cohort. Clin Infect Dis 2003;31:1170-4.

10. Lowy FD. Staphylococcus aureus infections. N Engl J Med 1998;339:520-32.

11. Willcox PA, Rayner BL, Whitelaw DA. Community-acquired Staphylococcus aureus bacteraemia in patients who do not abuse intravenous drugs. QJM 1998;91:41-7.

12. Sanabria TJ, Alpert JS, Goldberg R, Pape LA, Cheeseman SH. Increasing frequency of staphylococcal infective endocarditis: Experience at a university hospital, 1981 through 1988. Arch Intern Med 1990;150:1305-9.

13. Watanakunakorn C. Staphylococcus aureus endocarditis at a community teaching hospital, 1980 to 1991: An analysis of 106 cases. Arch Intern Med 1994;154:2330-5.

14. Fowler VG, Sanders LL, Kong LK, et al. Infective endocarditis due to Staphylococcus aureus: 59 prospectively identified cases with follow-up. Clin Infect Dis 1999;28:106-14.

15. Røder BL, Wandall DA, Frimodt-Møller N, et al. Clinical features of Staphylococcus aureus endocarditis: A 10-year experience in Denmark. Arch Intern Med 1999;159:462-9. 
16. Fang G, Keys TF, Gentry LO, et al. Prosthetic valve endocarditis resulting from nosocomial bacteremia: A prospective, multicenter study. Ann Intern Med 1993;119:560-7.

17. Mylonakis E, Calderwood SB. Infective endocarditis in adults. N Engl J Med 2001;345:1318-30.

18. Karchmer AW. Staphylococcal endocarditis. Laboratory and clinical basis for antibiotic therapy. Am J Med 1985;78(Suppl 6B):116-27.

19. Korzeniowski O, Sande MA, and The National Collaborative Endocarditis Study Group. Combination antimicrobial therapy for Staphylococcus aureus endocarditis in patients addicted to parenteral drugs and in nonaddicts: A prospective study. Ann Intern Med 1982;97:496-503

20. Watanakunakorn C, Baird IM. Prognostic factors in Staphylococcus aureus endocarditis and results of therapy with a penicillin and gentamicin. Amer J Med Sci 1977;273:133-9.

21. Abrams B, Sklaver A, Hoffman T, Greenman R. Single or combination therapy of staphylococcal endocarditis in intravenous drug abusers. Ann Intern Med 1979;90:789-91.

22. Chambers HF, Miller RT, Newman MD. Right-sided Staphylococcus aureus endocarditis in intravenous drug abusers: Two-week combination therapy. Ann Intern Med 1988;109:619-24.

23. Ribera E, Gómez-Jimenez J, Cortes E, et al. Effectiveness of cloxacillin with and without gentamicin in short-term therapy for right-sided Staphylococcus aureus endocarditis: A randomized, controlled trial. Ann Intern Med 1996;125:969-74.

24. Fortún J, Navas E, Martinez-Beltrán J. Short-course therapy for right-side endocarditis due to Staphylococcus aureus in drug abusers: Cloxacillin versus glycopeptides in combination with gentamicin. Clin Infect Dis 2001;33:120-5.

25. Ehni WF, Reller LB. Short-course therapy for catheterassociated Staphylococcus aureus bacteremia. Arch Intern Med 1989;149:533-6.
26. Raad II, Sabbagh MF. Optimal duration of therapy for catheterrelated Staphylococcus aureus bacteremia: A study of 55 cases and review. Clin Infect Dis 1992;14:75-82.

27. Jernigan JA, Farr BM. Short-course therapy of catheter-related Staphylococcus aureus bacteremia: A meta-analysis. Ann Intern Med 1993;119:304-11.

28. Malanoski GJ, Samore MH, Pefanis A, Karchmer A. Staphylococcus aureus catheter-associated bacteremia: Minimal effective therapy and unusual infectious complications associated with arterial sheath catheters. Arch Intern Med 1995:1161-6.

29. Zeylemaker MMP, Jaspers CAJJ, van Kraaij MGJ, Hoepelman VIM. Long-term infectious complications and their relation to treatment duration in catheter-related Staphylococcus aureus bacteremia. Eur J Clin Microbiol Infect Dis 2001;20:380-4.

30. Rosen AB, Fowler VG, Corey GR, et al. Cost-effectiveness of transesophageal echocardiography to determine the duration of therapy for intravascular catheter-associated Staphylococcus aureus bacteremia. Ann Intern Med 1999;130:810-20.

31. Fowler VG, Li J, Corey GR, et al. Role of echocardiography in evaluation of patients with Staphylococcus aureus bacteremia: Experience in 103 patients. J Am Coll Cardiol 1997;30:1072-8

32. Lodise TP, McKinnon PS, Swiderski L, Rybak MJ. Outcomes analysis of delayed antibiotic treatment for hospital-acquired Staphylococcus aureus bacteremia. Clin Infect Dis 2003;36:1418-23.

33. Blyth CC, Darragh H, Whelan A, et al. Evaluation of clinical guidelines for the management of Staphylococcus aureus bacteraemia. Intern Med J 2002;32:224-32.

34. Fowler VG, Sanders LL, Sexton DJ, et al. Outcome of Staphylococcus aureus bacteremia according to compliance with recommendations of infectious diseases specialists: Experience with 244 patients. Clin Infect Dis 1998;27:478-86. 


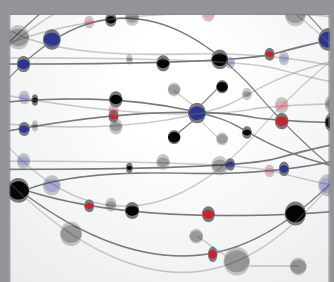

The Scientific World Journal
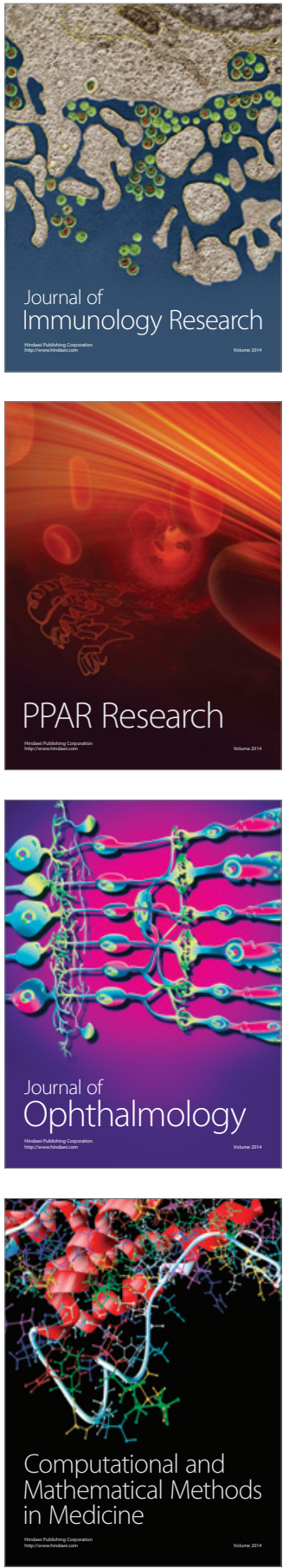

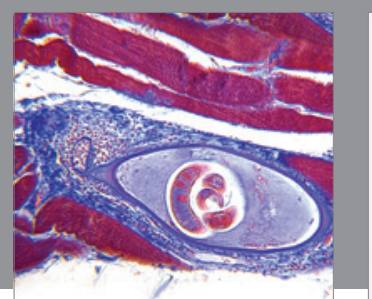

Gastroenterology Research and Practice

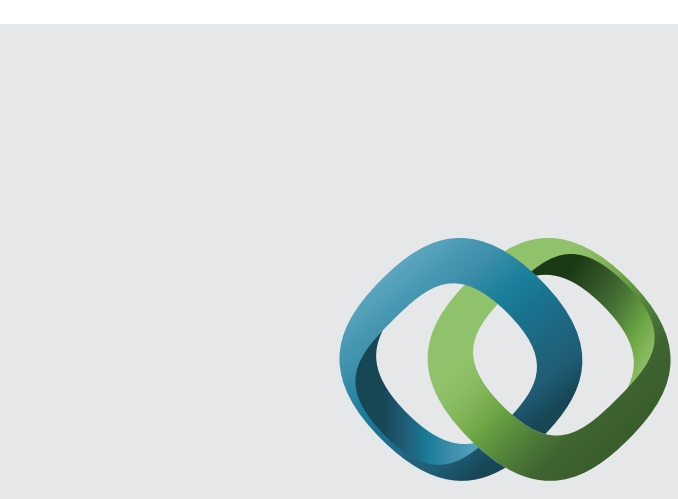

\section{Hindawi}

Submit your manuscripts at

http://www.hindawi.com
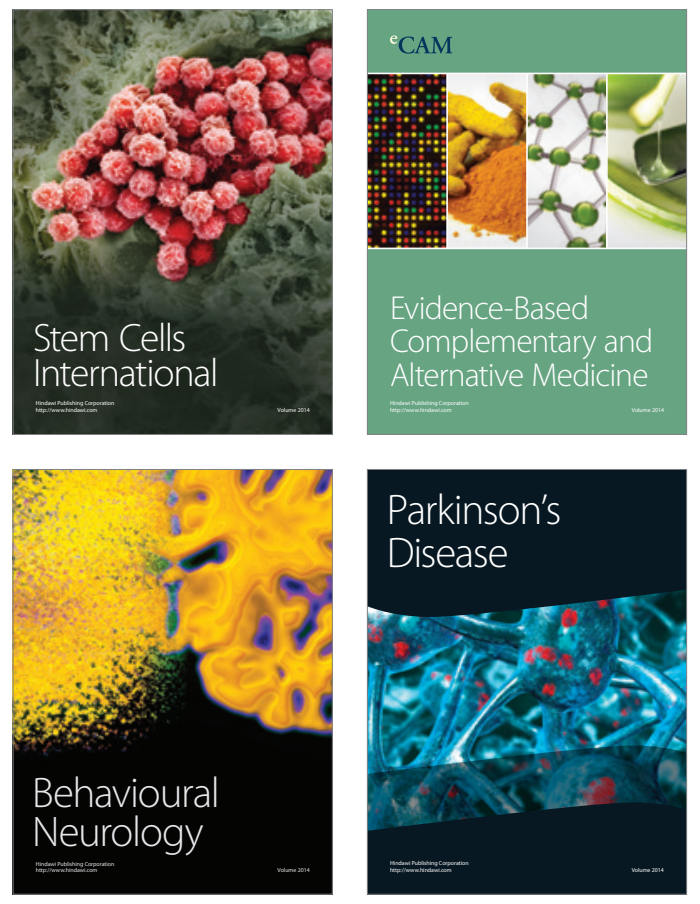
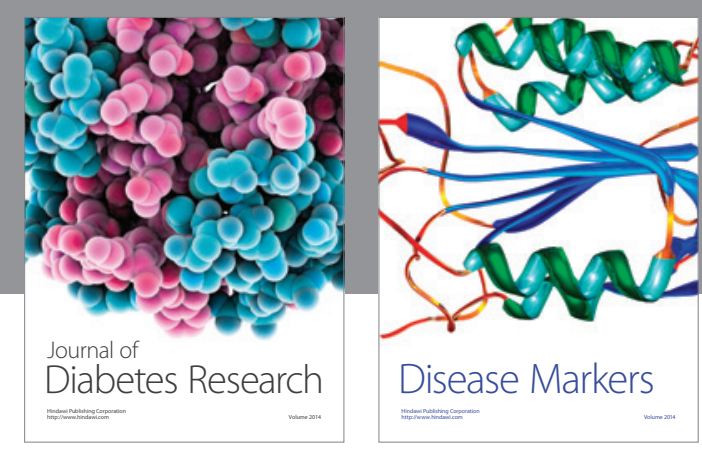

Disease Markers
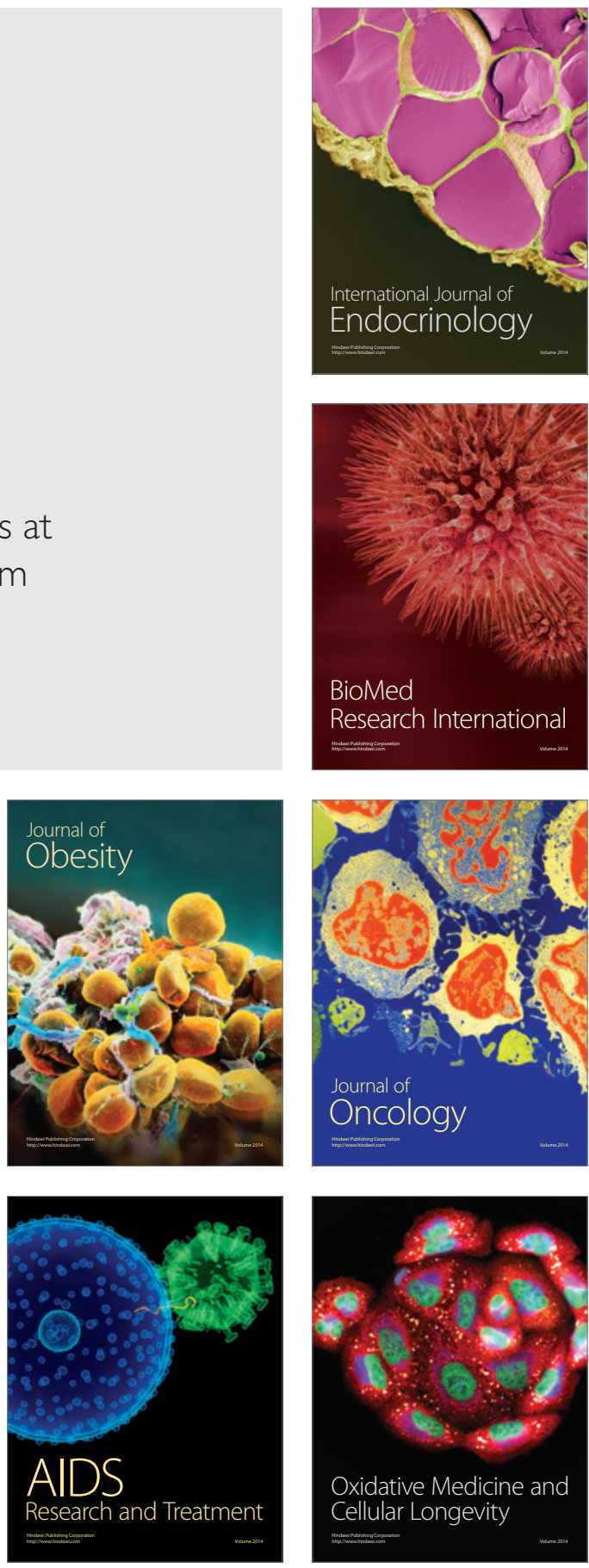